\title{
Research and Evaluation on Properties of High Temperature Seawater-based Fracturing Fluid
}

\author{
Wang Xingzun ${ }^{1}$, Guo Bumin ${ }^{1}$, Zheng Yan ${ }^{2}$, Bao Wenhui ${ }^{1}$, Bai Xiaodan², Chen Lei ${ }^{1}$, Sun \\ Houtai $^{1}$ \\ ${ }^{1}$ China Oilfield Services Ltd., Tianjin 300450, China \\ ${ }^{2}$ Beijing AP Polymer Technology Company Ltd.,Beijing 102200,China
}

Keywords: seawater-based; fracturing fluid; high temperature; quick solvent and salt tolerance; high temperature resistance and high shear resistance; low damage

\begin{abstract}
In order to make up for the lack of high temperature resistance, the high temperature seawater-based fracturing fluid with temperature $180^{\circ} \mathrm{C}$ is developed with the self-developed high temperature seawater based frac agent HYCF-5 and its supporting seawater-based reversible cross joint agent HYCF-B, efficient chelating agent HYCF-S, high temperature stabilizer HYCF-C, performance regulator HYCF-D, anti-swelling agent HYAS-1, cleanup additive HYCF-F. Through the performance evaluation of the high temperature seawater-based fracturing fluid, the performance evaluation of high temperature seawater-based fracturing fluid and the performance research and evaluation of the high temperature seawater-based fracturing fluid, the correlation can be met by the standard of oil and gas industry standard SY/T 6376-2008 the technical conditions of fracturing liquid. At the same time, the paper shows the high suspension sand advantage and low damage mechanism of high temperature seawater-based fracturing fluid, and provides sufficient theoretical reference and technical support for the field application.
\end{abstract}

\section{Foreword}

In recent years, with the in-depth strategic development of oil and gas resources, the ocean has become one of the important trends to developing oil and gas resources since the offshore oil and gas resources are extremely abundant. As an important method for sand exclusion and yield increase, offshore fracturing is employed increasingly. Based on the offshore platform, the high temperature seawater-based fracturing fluid system is designed to realize continuous mixing, so as to lower the cost, improve the working efficiency, save the fresh water resource and accomplish high-efficiency development of offshore oil and gas fields ${ }^{[1]}$.

At present, the thickening agent researched and developed at home and abroad for high temperature seawater-based fracturing fluid includes three types, i.e. natural polymers such as guanidine gum and modified guanidine gum, viscoelastic surfactant and synthetic polymer ${ }^{[2-7]}$. In these days, the crosslinked gel fracturing fluid containing natural polymers such as guanidine gum and modified guanidine gum as the thickening agent has been researched to the great extent. This type of fracturing fluid can resist up to $160^{\circ} \mathrm{C}$, which has been applied in the oil fields, exhibiting excellent properties. Since the viscoelastic surfactant fracturing fluid is used with high concentrate at low temperature and high cost, it is unsuitable for high temperature oil and gas reserves, so that its application and development are limited in the high temperature seawater-based oil and gas reserves. By introducing special functional groups, the synthetic polymer fracturing fluid improves the swelling properties, temperature resistance, salt resistance and shear resistance of the polymer. Since the synthetic polymer functional groups and molecular weight are controllable, synthesis and application of the polymer containing special functional groups and formed with special crosslinking method, it is expected that such polymer could meet the requirement of resisting $180^{\circ} \mathrm{C}$ and even higher. Therefore, the instant $180^{\circ} \mathrm{C}$ resisting seawater-based fracturing fluid is the research direction of new type seawater-based fracturing fluid. In this paper, the independently developed high temperature seawater-based fracturing fluid thickening agent HYCF-5, its 
supportive seawater-based reversible crosslinker HYCF-B, and additives, including the efficient chelating agent HYCF-S, the high-temperature stabilizing agent HYCF-C, the property conditioning agent HYCF-D, the antiswelling agent HYAS-1 and the cleanup additive HYCF-F, are used to prepare the $180^{\circ} \mathrm{C}$ resisting high temperature seawater-based fracturing fluid. In addition, research and evaluation is performed on properties of the high temperature seawater-based fracturing fluid thickening agent, high temperature seawater-based fracturing fluid and high temperature seawaterbased fracturing fluid gel breaker, thereby providing adequate theoretical reference and technical support for field application of high temperature seawater-based fracturing fluid.

\section{Experiment}

High temperature seawater-based fracturing fluid thickening agent HYCF-5, seawater-based reversible crosslinker HYCF-B, efficient chelating agent HYCF-S, high-temperature stabilizing agent HYCF-C, property conditioning agent HYCF-D, antiswelling agent HYAS-1 and discharge aiding agent HYCF-F, self-made; potassium chloride, potassium sulfate, ammonium persulfate and analytical pure produced by Tianjin Guangfu Technology Development Co., Ltd.; sodium chloride and analytical reagent produced by Beijing Chemical Works; anhydrous magnesium chloride, anhydrous magnesium sulfate and analytical pure manufactured by Tianjin Guangfu Fine Chemical Research Institute; calcium sulphate dihydrate, anhydrous calcium chloride and analytical reagent made by Tianjin Fuchen Chemical Reagent Factory; magnesium bromide hexahydrat and chemical pure produced by Shanghai Aladdin Bio-Chem Technology Co., Ltd.; deionized water with conductivity $\leq 1 \mathrm{uS} / \mathrm{cm}$, self-made; anhydrous kerosene and analytical pure by Tianjin BASF Chemical Co., Ltd.; experimental sodium bentonite by Xinjiang Nonmetallic Minerals Xiazijie Bentonite Co., Ltd.; ceramsite proppant, 20-40 mesh, Kabo Ceramsite Co., Ltd.; artificial core with gas permeability 10-100×10-3um2, made by Beijing Jiadeyibang Petroleum Technology Co., Ltd.

S312 Digital Display Constant Speed Mixer, by Shanghai SENCO Technology Co., Ltd.; RS6000 Haake Rheometer, by Haake; ZNN-D6S Digital Display Six-Speed Spinning Viscosimeter and GRL-BX Portable Roller Heating Furnace, by Qingdao BAIRUIDA Petroleum Machinery Manufacturing Co., Ltd.; JYW-200B Automatic Interface Tension Gauge, by Chengde Baohui Testing Machine Co., Ltd.; nanometer particle size and Zeta potentiometer, by Malvern Panalytical Co., Ltd.; SL200KB Optical Method Contact Angle/Interface Tension Gauge, by Solon (Shanghai) Information Technology Co., Ltd.; GGS42-2 High-Temperature and High-Pressure Filter Press, Qingdao Yize Electromechanical Technology Co., Ltd.; MZ-IV Fracturing Fluid Friction Tester, and High-Temperature and High-Pressure Core Dynamic Filter Press, by Haian Oil Scientific Research Apparatus Co., Ltd.; large visual combination fissure proppant migration and laying simulation experiment apparatus, by China University of Petroleum (East China); fracturing fluid dynamic leak-off damage test system, by China Oilfield Services Limited; FEI Quanta 200F Field Emission Environmental Scanning Electron Microscope, by FEI; and DAWN HELEOS Gel Permeation Chromatograph, by Wyatt Technology Corporation.

After studying the content of main salts in the seawater as referred in the book Preparation and Application of Oilfield Chemicals published by Chemical Industry Press, the formula of simulated seawater is as listed in Table 1 (the total salinity of simulated seawater is $34910 \mathrm{mg} / \mathrm{L}$ )[8]. For the simulated seawater to be used in the experiments hereinafter, this formula is adopted together with deionized water for solution preparation.

Table 1 Formula of Simulated Seawater

\begin{tabular}{ccc}
\hline Component & Content of salt in 100g of seawater $(\mathrm{g})$ & Proportion in total salt content (\%) \\
\hline $\mathrm{NaCl}$ & 2.7123 & 77.8 \\
$\mathrm{MgCl}_{2}$ & 0.3807 & 10.9 \\
$\mathrm{MgSO}_{4}$ & 0.1658 & 4.7 \\
$\mathrm{CaSO}_{4}$ & 0.1260 & 3.6 \\
$\mathrm{~K}_{2} \mathrm{SO}_{4}$ & 0.0863 & 2.5 \\
$\mathrm{CaCl}_{2}$ & 0.0123 & 0.3 \\
$\mathrm{MgBr}_{2}$ & 0.0076 & 0.2 \\
\hline
\end{tabular}


Formula and Preparation Method of High Temperature Seawater-based Fracturing Fluid

Formula of high temperature seawater-based fracturing fluid: 1.0\% HYCF-5+2.5\% HYCF-S $+0.2 \%$ HYCF-C+0.5\% HYCF-D+0.2\% HYCF-F+0.5\% HYAS-1+0.6\% HYCF-B.

Preparation method: to the simulated seawater successively add 2.5\% (mass fraction, the same below) of HYCF-S, $0.2 \%$ of HYCF-C, $0.2 \%$ of HYCF-F, $0.5 \%$ of HYAS- 1 and $0.5 \%$ of HYCF-D, and then add $1.0 \%$ of HYCF-5, stir for 5 min to obtain the matrix of high temperature seawaterbased fracturing fluid. Add $0.6 \%$ of HYCF-B in the matrix for crosslinking to obtain the high temperature seawater-based fracturing fluid.

Evaluation Method for Instant Dissolution of High Temperature Seawater-based Fracturing Fluid Thickening Agent HYCF-5

Prepare the high temperature seawater-based fracturing fluid according to the method detailed in 2.2.2. In the preparation process, test the matrix viscosity after stirring $1.0 \%$ of HYCF-5 for 1 min, $2 \mathrm{~min}$, 3min, $4 \mathrm{~min}$ and $5 \mathrm{~min}$ and standing for $30 \mathrm{~min}$.

Evaluation Method for Resistance Reduction of High Temperature Seawater-based Fracturing Fluid Thickening Agent HYCF-5

The viscosity of $0.35 \%$ of HYCF-5 prepared with fresh water is $30-50 \mathrm{mPa} \cdot \mathrm{s}$, basically equal to that of high temperature seawater-based fracturing fluid at $180^{\circ} \mathrm{C}$. The resistance reduction effect of $0.35 \%$ of HYCF-5 is assessed at room temperature by referring to the standard in the oil and gas industry SY/T 5107-2005 Recommended Practices on Measuring the Properties of Water-Based Fracturing Fluid.

Evaluation Method for Temperature Resistance, Shear Resistance and Viscoelasticity of High Temperature Seawater-based Fracturing Fluid

Prepare the high temperature seawater-based fracturing fluid described in the formula described in 2.2.2, and test on relevant parameters by referring to the standard in the oil and gas industry SY/T 5107-2005 Recommended Practices on Measuring the properties of Water-Based Fracturing Fluid.

Test Method for Rheological Parameters of High Temperature Seawater-based Fracturing Fluid

Prepare the high temperature seawater-based fracturing fluid with the method described in 2.2.2 and test its variable shear property at $180^{\circ} \mathrm{C}$ with the Haake Rheometer, where, $1 \#$ is ladder-like variable shear, 2\# is linear variable shear and the rate of shear is in the range of 100-511s-1. According to the variable shear rheological curve, $\mathrm{n}^{\prime}$ value and $\mathrm{K}^{\prime}$ value are calculated by referring to the standard in the oil and gas industry SY/T 5107-2005 Recommended Practices on Measuring the Properties of Water-Based Fracturing Fluid.

Prepare the high temperature seawater-based fracturing fluid according to the method described in 2.2.2, stir the fluid for $5 \mathrm{~min}$ and then add $30 \%$ (volume fraction) of ceramsite; after stirring evenly, add $0.6 \%$ (mass fraction) of HYCF-B; after crosslinking, pour in the measuring cylinder to observe the solid-carrying behavior.

Compare the migration and laying of proppant in different sand-carrying fluids by using the large visual combination fracture proppant migration and laying simulation experiment unit, so as to evaluate the dynamic sand-carrying effect[9]. The proppant particle size is 20/40 meshes, density $2.6 \mathrm{~g} / \mathrm{cm} 3$, sand proportion $10 \%$ and displacement $5.4 \mathrm{~m} 3 / \mathrm{h}$, and at the same time, assess the hydroxypropyl guanidine gum matrix, high temperature seawater-based fracturing fluid and crosslinked fluid. See Table 2 for the experimental formula.

Table 2 Formula of Fracturing Fluid in the Dynamic Sand-Carrying Experiment

\begin{tabular}{ccc}
\hline No. & Formula of fracturing fluid & Viscosity, mPa·s \\
\hline 1 & Fresh water & 1 \\
2 & $0.375 \%$ HPG & 40 \\
3 & $0.35 \%$ HYCF-5 & 30 \\
4 & $0.35 \%$ HYCF-5 & 80 \\
\hline
\end{tabular}

Evaluation Method for Static Filtration Property, Dynamic Filtration Property and Dynamic Filtration Permeability Loss Rate of High Temperature Seawater-based Fracturing Fluid 
Prepare the high temperature seawater-based fracturing fluid described in the formula described in 2.2.2, and test its properties by referring to the standard in the oil and gas industry SY/T 51072005 Recommended Practices on Measuring the Properties of Water-Based Fracturing Fluid.

Gel Breaking Method of High Temperature Seawater-based Fracturing Fluid

Prepare the high temperature seawater-based fracturing fluid described in the method described in 2.2 .2 , add $0.3 \%$ (mass fraction) of ammonium persulfate, place in the high temperature aging tank for hot aging gel breaking at $180^{\circ} \mathrm{C}$ for $8 \mathrm{~h}$, to obtain gel breaker after cooling to room temperature.

Evaluation Method for Gel Breaker Interfacial Tension, Residue Content and Core Matrix Permeability Loss Rate of High Temperature Seawater-based Fracturing Fluid

Take the gel breaker of high temperature seawater-based fracturing fluid prepared described in the formula described in 2.2.2, and test the properties thereof by referring to the standard in the oil and gas industry SY/T 5107-2005 Recommended Practices on Measuring the Properties of WaterBased Fracturing Fluid.

Evaluation Method for Molecular Weight and Its Distribution of Gel Breaker for High Temperature Seawater-based Fracturing Fluid

Take the gel breaker of high temperature seawater-based fracturing fluid mentioned in 2.3.8, measure its molecular weight and distribution with the gel permeation chromatography (GPC) by referring to the national standard GB/T 21863-2008 Gel Permeation Chromatography (GPC) - Part 1: Tetrahydrofuran (THF) as Elution Solvent.

Evaluation Method for Micelle Particle Size of Gel Breaker for High Temperature Seawaterbased Fracturing Fluid

Take the gel breaker for the high temperature seawater-based fracturing fluid mentioned in 2.3.8, and measure the micelle particle size of gel breaker with the nanometer particle size and Zeta measuring apparatus.

Place the ceramsite under the condition of $105^{\circ} \mathrm{C}$ and dry to constant weight, and cool to room temperature for use. Measure the ceramsite at 20\% (volume fraction) and weigh its mass, and then vacuumize the ceramsite in the simulated seawater and gel breaker for further saturation, dry at $105^{\circ} \mathrm{C}$ to constant weight and then weigh the ceramsite, and calculate the adsorbing capacity of ceramsite in the simulated seawater and gel breaker ${ }^{[10]}$. Scan ceramsite 1 (dried but not adsorbing), ceramsite 2 (adsorbing simulated seawater) and ceramsite 3 (adsorbing gel breaker) the environmental scanning electron microscope and analyze the difference of ceramsite adsorbing simulated seawater and gel breaker ${ }^{[11]}$.

\section{Result and Discussion}

Evaluation on the Properties of High Temperature Seawater-based Fracturing Fluid Thickening Agent HYCF-5

Instant Dissolution of High Temperature Seawater-based Fracturing Fluid Thickening Agent HYCF-5

Prepare the high temperature seawater-based fracturing fluid described in the method described in 2.2.2, and test the matrix viscosity of thickening agent for the high temperature seawater-based fracturing fluid. The matrix viscosity is around $100 \mathrm{mPa} \cdot \mathrm{s}$ after stirring for $5 \mathrm{~min}$ and standing for $30 \mathrm{~min}$, which meets the requirements of field continuous mixing for instant dissolution and pumping.

Table 3 Matrix Viscosity and Sand-Carrying Behavior of HYCF-5

\begin{tabular}{ccccccc}
\hline Stirring time/min & 1 & 2 & 3 & 4 & 5 & Standing for 30 \\
Viscosity/(mPa $\mathrm{s})$ & 15.6 & 24.9 & 40.5 & 61.5 & 102.0 & 105.0 \\
\hline
\end{tabular}

Resistance Reduction of High Temperature Seawater-based Fracturing Fluid Thickening Agent HYCF-5

Test the resistance reduction property of HYCF-5 described in 2.3.2, measure the differential 
pressure at the displacements of $39 \mathrm{~L} / \mathrm{min}, 52 \mathrm{~L} / \mathrm{min}$ and $65 \mathrm{~L} / \mathrm{min}$, and then calculate the resistance reduction ratio, as shown in Table 4. According to the table, the resistance reduction ratio of HYCF5 under three different displacements reach more than 50\%, indicating HYCF-5 has a good resistance reduction effect.

Table 4 Resistance Reduction Ratio at Different Experimental Flow Rates

\begin{tabular}{ccccc}
\hline $\begin{array}{c}\text { Experimental } \\
\text { displacement/(L/min) }\end{array}$ & $\begin{array}{c}\text { Experimental } \\
\text { flow rate/(m/s) }\end{array}$ & $\begin{array}{c}\text { Fresh water } \\
\text { differential } \\
\text { pressure/KPa }\end{array}$ & $\begin{array}{c}\text { Differential } \\
\text { pressure of } \\
0.35 \% \text { HYCF- } \\
5 / \text { KPa }\end{array}$ & $\begin{array}{c}\text { Resistance } \\
\text { reduction ratio of } \\
0.35 \% H Y C F-5 / \%\end{array}$ \\
\hline 65.0 & 13.8 & 434 & 163.6 & 62.30 \\
52.0 & 11.0 & 287 & 115 & 59.93 \\
39.0 & 8.3 & 163 & 75.8 & 53.50 \\
\hline
\end{tabular}

Evaluate the temperature resistance and shear resistance of high temperature seawater-based fracturing fluid according to 2.3.3. For the rheological shear curve of high temperature seawaterbased fracturing fluid at $180^{\circ} \mathrm{C}$, see Figure 1.

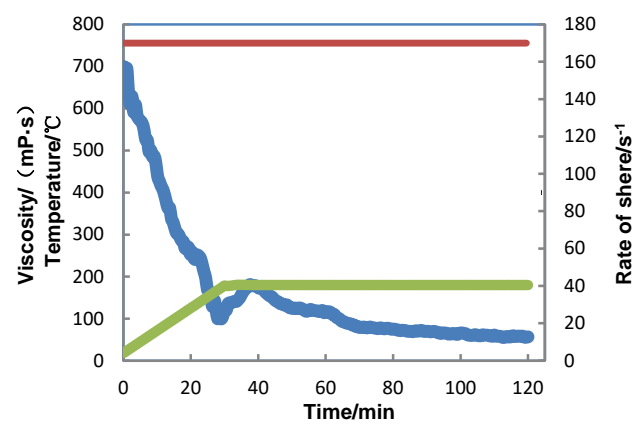

Figure 1 Rheological Curve of High Temperature Seawater-based Fracturing Fluid

The viscosity of high temperature seawater-based fracturing fluid is $56.70 \mathrm{mPa} \cdot \mathrm{s}$ after subjecting to shear at $180^{\circ} \mathrm{C}$. As shown in the figure, its viscosity decreases when the temperature rises. After it reaches $180^{\circ} \mathrm{C}$, the viscosity remains stable basically and finally reaches more than $50 \mathrm{mPa} \cdot \mathrm{s}$, showing good temperature resistance and shear resistance properties.

Evaluate the viscoelasticity of high temperature seawater-based fracturing fluid described in 2.3.5. After determining the linear viscoelastic range of fracturing fluid below $30^{\circ} \mathrm{C}$, scan the oscillation time to obtain the relation curve of $t-G^{\prime} / G^{\prime \prime}, G^{\prime}=40.85 P a$ and $G$ " $=10.04 \mathrm{~Pa}, G^{\prime}$ and $\mathrm{G}^{\prime}$ " meet the industrial indicators $\mathrm{G}^{\prime} \geq 1.5 \mathrm{~Pa}$ and $\mathrm{G}^{\prime \prime} \geq 0.3 \mathrm{~Pa} . \mathrm{G}^{\prime}$ of fracturing fluid is greater than $G$ " , G' / G " =4.07, showing good elasticity. The sand-carrying behavior of fracturing fluid is related to both system viscosity and its elasticity, and its elasticity contributes more to the sandcarrying behavior of fracturing fluid ${ }^{[12]}$. The high temperature seawater-based fracturing fluid belongs to a viscoelastic polymer fluid.

Test the rheological parameters of high temperature seawater-based fracturing fluid described in 2.3.4. For rheological curves of $1 \#$ and 2\# variable shears, see Figures 2 and 3.
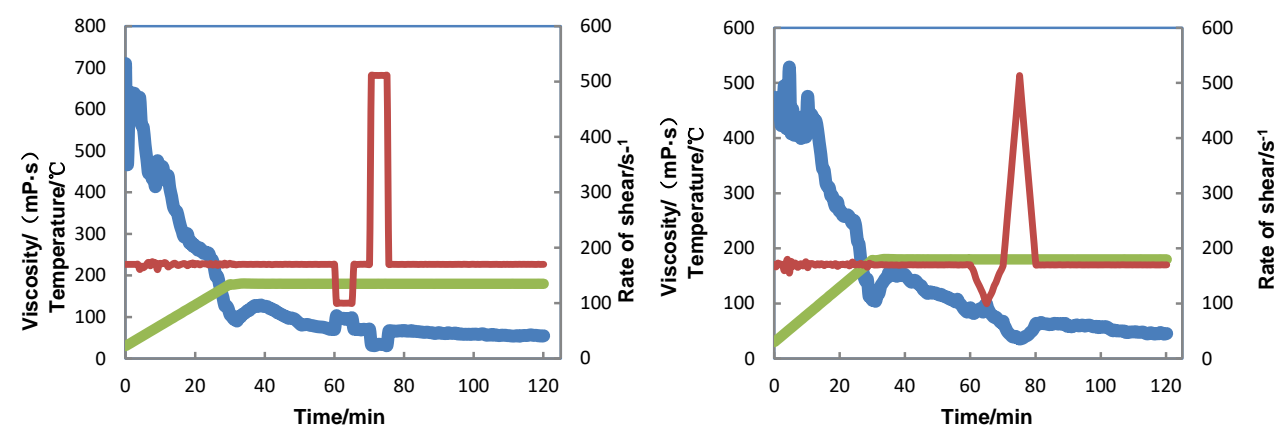

Figure 2 Rheological Curve of 1\# Variable Shear Figure 3 Rheological Curve of 2\# Variable Shear 
According to the variable shear test data and rheological curves, treat and analyze the results, as listed in Table 5. It is shown that the shear recovery rate is high after variable shear, indicating the shear recovery of high temperature seawater-based fracturing fluid is good, while the shear recovery rate of conventional polymer is below $33 \%$ and the conventional polymer shear is unrecoverable ${ }^{[13]}$. According to $\mathrm{n}^{\prime}$ and $\mathrm{K}^{\prime}$, the high temperature seawater-based fracturing fluid is a pseudoplastic fluid, exhibiting excellent thickening ability and anti-shear ability.

Table 5 Analysis and Result of Rheological Parameters

\begin{tabular}{cccccc}
\hline No. & $\begin{array}{c}\text { Shear viscosity } \\
\text { 170s-1 before } \\
\text { variable } \\
\text { shear/(mPa } \mathrm{s})\end{array}$ & $\begin{array}{c}\text { Shear viscosity } \\
170 \mathrm{~s}^{-1} \text { after variable } \\
\text { shear/(mPa·s) }\end{array}$ & $\begin{array}{c}\text { Shear recovery } \\
\text { rate/\% }\end{array}$ & $\begin{array}{c}\text { Rheological } \\
\text { behavior } \\
\text { index n' }\end{array}$ & $\begin{array}{c}\text { Consistency } \\
\text { coefficient K' } \\
/\left(\mathrm{mPa} \cdot \mathrm{sn}^{\prime}\right.\end{array}$ \\
\hline 1 & 70.43 & 54.78 & 78 & 0.3176 & 2.3823 \\
2 & 86.50 & 45.55 & 53 & 0.3059 & 2.6330 \\
\hline
\end{tabular}

Evaluate the static sand-carrying capability of high temperature seawater-based fracturing fluid described in 2.3.5. Through testing, the static sand-carrying time of high temperature seawaterbased fracturing fluid is more than $60 \mathrm{~min}$, indicating the static sand-carrying behavior of fracturing fluid is good.

Dynamic Sand-Carrying Capability of High Temperature Seawater-based Fracturing Fluid

Evaluate the dynamic sand-carrying capability of high temperature seawater-based fracturing fluid described in 2.3.6. Select and process the photos of proppants with different formulas that are taken with visual tablet PC in the process of migration, as shown in Figure 4.
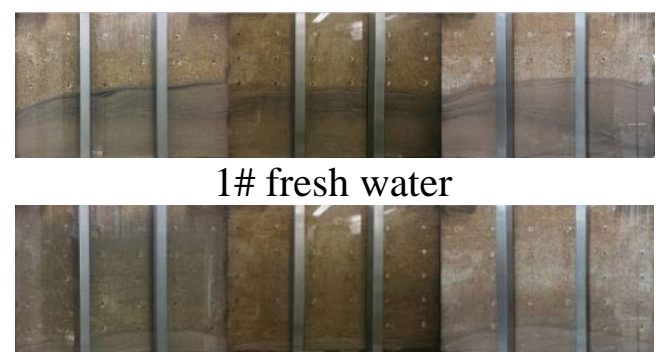

2\# $0.375 \% H P G$

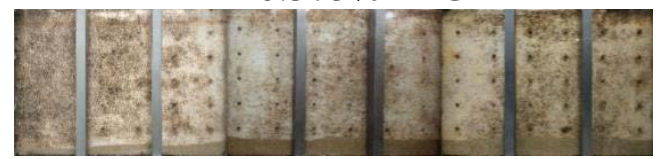

3\# $0.35 \% H Y C F-5$

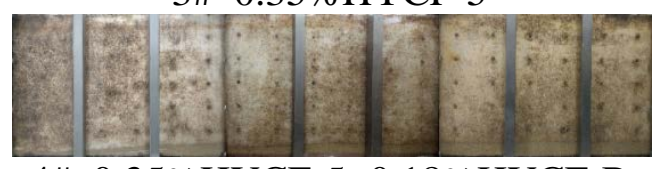

4\# $0.35 \% H Y C F-5+0.18 \% H Y C F-B$

Figure 4 Migration Effect of Different Proppants for Fracturing Fluid

As shown in Figure 4, comparing the experiment on $3 \#$ and $4 \#$ to that on $1 \#$ and $2 \#$, the sand levee balance height decreases significantly, indicating the dynamic sand-carrying capacity of HYCF-5 matrix and crosslinking agent improves substantially over the conventional guanidine gum fracturing fluid. Furthermore, the sand levee height of $4 \#$ is slightly lower than that of $3 \#$, indicating the crosslinking fracturing fluid is of greater viscosity, the proppant settles less in the pipeline and its dynamic sand-carrying capacity is better.

Evaluate the static filtration property of high temperature seawater-based fracturing fluid described in 2.3.7. The rest results are shown in Table 6 after testing the static filtration and processing the data. According to the table, the static filtration property of high temperature seawater-based fracturing fluid meets the indicator specified in the industrial standard. 
Table 6 Static Filtration Property of High Temperature Seawater-based Fracturing Fluid

\begin{tabular}{cccc}
\hline Item & $\begin{array}{c}\text { C3 filtration } \\
\text { coefficient } /(-*)\end{array}$ & $\begin{array}{c}\text { Qsp spurt loss } \\
\text { volume } /\left(\mathrm{m}^{3} / \mathrm{m}^{2}\right)\end{array}$ & $\begin{array}{c}\text { Vc filtration } \\
\text { rate/(m/min })\end{array}$ \\
\hline $\begin{array}{c}\text { Indicator } \\
\text { High temperature seawater- } \\
\text { based fracturing fluid }\end{array}$ & $0.1 .0^{*} 10^{-3}$ & $\leq 5.0^{*} 10^{-2}$ & $\leq 1.5^{*} 10^{-4}$ \\
\hline
\end{tabular}

Evaluate the dynamic filtration property of high temperature seawater-based fracturing fluid described in 2.3.7. The rest results are shown in Table 7 after testing the static filtration and processing the data. According to the table, the dynamic filtration property of high temperature seawater-based fracturing fluid meets the indicator specified in the industrial standard.

Table 7 Dynamic Filtration Property of High Temperature Seawater-based Fracturing Fluid

\begin{tabular}{cccc}
\hline Item & $\begin{array}{c}\text { C3 filtration } \\
\text { coefficient } /(* \sqrt[m i n]{\min })\end{array}$ & $\begin{array}{c}\text { Qsp spurt loss } \\
\text { volume } /\left(\mathrm{m}^{3} / \mathrm{m}^{2}\right)\end{array}$ & $\begin{array}{c}\text { Vc filtration } \\
\text { rate } /(\mathrm{m} / \mathrm{min})\end{array}$ \\
\hline $\begin{array}{c}\text { Indicator } \\
\text { High temperature seawater- } \\
\text { based fracturing fluid }\end{array}$ & $0.4 .0 \times 10^{-3}$ & $\leq 5.0 \times 10^{-2}$ & $\leq 1.5 \times 10^{-3}$ \\
\hline
\end{tabular}

Evaluate the dynamic filtration permeability loss rate of high temperature seawater-based fracturing fluid described in 2.3.7; the experimental temperatures are $25^{\circ} \mathrm{C}$ and $90^{\circ} \mathrm{C}$ and the test results are shown in Table 8 . The test results meet the indicator requirement: dynamic filtration permeability loss rate $\leq 60 \%$, indicating the dynamic filtration permeability loss rate of high temperature seawater-based fracturing fluid is low and beneficial to improvement in yieldincreasing effect after fracturing.

Table 8 Dynamic Filtration Permeability Loss Rate of High Temperature Seawater-based Fracturing Fluid

\begin{tabular}{ccccc}
\hline Core & \multicolumn{2}{c}{ Permeability $/\left(10^{-3} \mathrm{um}^{2}\right)$} & \multirow{2}{*}{ Loss rate $/ \%$} & Experimental temperature $/{ }^{\circ} \mathrm{C}$ \\
\cline { 2 - 3 } No. & Before loss & After loss & & 25 \\
\hline 1 & 0.3326 & 0.2615 & 21.38 & 90 \\
\hline
\end{tabular}

Research and Evaluation on the Properties of Gel Breaker for High Temperature Seawater-based Fracturing Fluid

Break the gel of high temperature seawater-based fracturing fluid described in 2.3.8. At room temperature, the gel breaker is faint yellow slightly transparent liquid and its measured viscosity is $3.3 \mathrm{mPa} \cdot \mathrm{s}$ the fracturing fluid gel is broken completely.

Take the gel breaker of high temperature seawater-based fracturing fluid described in 2.3.8 to test the surface and interfacial tension and contact angle, as shown in Table 9. As for the gel breaker, its surface and interfacial tension is lower, and its contact angle declines compared to that of deionized water $\left(50.956^{\circ}\right)$, indicating the gel breaker is of some wettability.

Table 9 Surface and Interfacial Tension and Wetting Angle of Gel Breaker

\begin{tabular}{cccc}
\hline Gel breaker & $\begin{array}{c}\text { Surface tension/ } \\
\left(\mathrm{mN} \cdot \mathrm{m}^{-1}\right)\end{array}$ & $\begin{array}{c}\text { Interfacial } \\
\text { tension } /\left(\mathrm{mN} \cdot \mathrm{m}^{-1}\right)\end{array}$ & ${\text { Contact angle }{ }^{\circ}}^{\text {Indicator }}$ \\
\hline 28 & $\leq 2$ & $\begin{array}{c}37.911 \text { (deionized } \\
\text { water) }\end{array}$ \\
$\begin{array}{c}\text { High temperature seawater- } \\
\text { based fracturing fluid }\end{array}$ & 27.38 & 1.63 & 31.722 \\
\hline
\end{tabular}

Take the gel breaker for high temperature seawater-based fracturing fluid described in 2.3.8 to measure the residue content as $45.0 \mathrm{mg} / \mathrm{L}$, indicating the residue content of gel breaker is very low, 
much lower than the industrial requirement of $600 \mathrm{mg} / \mathrm{L}$.

Take the gel breaker for high temperature seawater-based fracturing fluid described in 2.3.8, measure its weight-average molecular weight of $29.6 \times 104$ and number-average molar mass of $18.46 \times 104$. For the gel breaker for guanidine gum fracturing fluid, the weight-average molecular weight is $151.9 \times 104$ (gel breaking for $4 \mathrm{~h}$ ) and number-average molar mass is $124.2 \times 104$ (gel breaking for $24 \mathrm{~h}$ ), being 4-5 times that of the gel breaker for high temperature seawater-based fracturing fluid[14]. The lower the molecular weight, more completely is degraded the polymer, and the more difficult for the low polymer and its derivative to form local secondary weak crosslinking with the crosslinking agent, so as to keep cleanliness and low loss effect of gel breaker.

Take the gel breaker for high temperature seawater-based fracturing fluid described in 2.3.8, and measure the micelle particle size Z-Average of $0.3454 \mathrm{um}$. For the gel breaker for guanidine gum fracturing fluid, the micelle particle size is 93.86um (gel breaking for 4h) and 89.09um (gel breaking for 24h), being more than 200 times that of the gel breaker for high temperature seawaterbased fracturing fluid[14]. The lower the particle size, the easier for it to flow in the porous medium, to reduce blocking to the prop fracture and lessen damage to the strata.

Through experiment, the adsorbing capacity of ceramsite to the simulated seawater is $0.0094 \mathrm{~g} / \mathrm{g}$, while that to the gel breaker is $0.0139 \mathrm{~g} / \mathrm{g}$, up by $48 \%$. The adsorption of ceramsite to the simulated seawater is salts adsorption, while that to the gel breaker includes salt adsorption and other adsorption; the former is adsorption of a dominantly large amount of salt, while the latter is mainly adsorption of the residual low-molecule aggregate and surfactant.

As shown in Figure 5 are $20000 \times$ pictures of ceramsite 1, 2 and 3 that are enlarged with the environmental scanning electron microscope. According to the pictures, the surface texture of ceramsite 1 is neat and consistent without visible attachment, that of ceramsite 2 is attached with some white granular substance, which is the crystal separating out from the salt on the ceramsite surface; and besides white granular substance, that of ceramsite 3 exhibits some bumps and irregular matters, which are basically identical to the residual low-molecule aggregation and surfactant identified in the theoretical analysis.
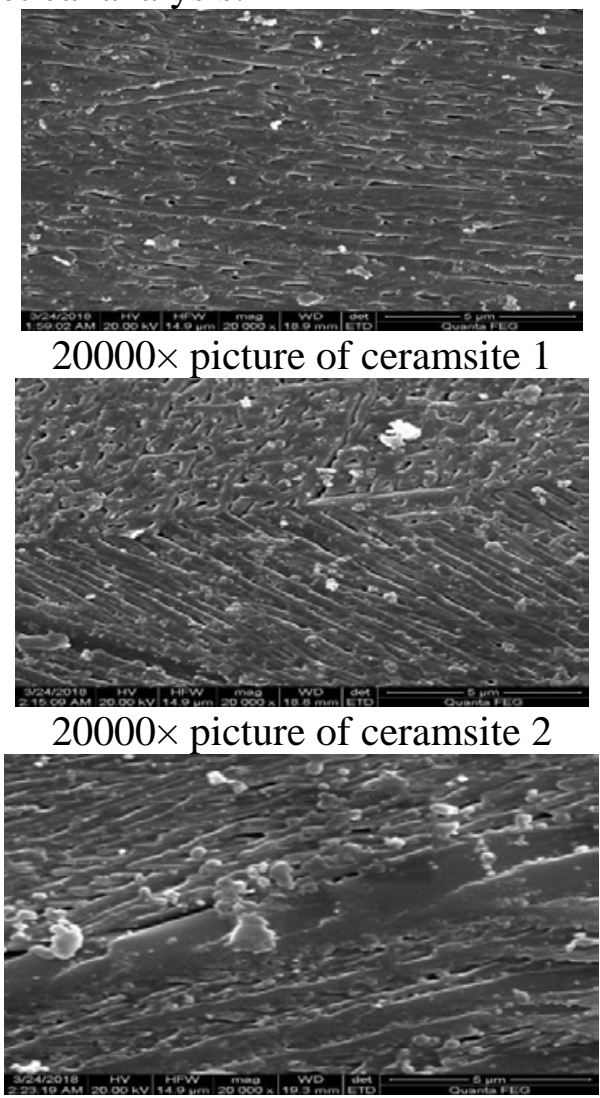

20000× picture of ceramsite 3

Figure 5 Change in Surface Features after Adsorption of Ceramsite to Gel Breaker 
Take the gel breaker for high temperature seawater-based fracturing fluid described in 2.3.8 to test its core matrix permeability loss rate, as shown in Table 10. For the conventional guanidine gum fracturing fluid, the reservoir matrix permeability loss rate is $20-30 \%{ }^{[15]}$, which is far lower than that of the high temperature seawater-based fracturing fluid. The high temperature seawaterbased fracturing fluid features low loss, even no loss, beneficial to yield-increasing effect after fracturing.

Table 10 Gel Breaker Core Matrix Permeability Loss Rate

\begin{tabular}{cccc}
\hline \multirow{2}{*}{ Core No. } & \multicolumn{2}{c}{ Permeability $/\left(10^{-3} \mathrm{um}^{2}\right)$} & \multirow{2}{*}{ Loss rate/\% } \\
\cline { 2 - 3 } & Before loss & After loss & \\
\hline 1 & 0.1810 & 0.1751 & 3.25 \\
2 & 0.1798 & 0.1862 & -3.56 \\
\hline
\end{tabular}

\section{Conclusion}

In this paper, the independently developed high temperature seawater-based fracturing fluid thickening agent HYCF-5, its supportive seawater-based reversible crosslinker HYCF-B, and additives, including the efficient chelating agent HYCF-S, the high-temperature stabilizing agent HYCF-C, the property conditioning agent HYCF-D, the antiswelling agent HYAS-1 and the cleanup additive $\mathrm{HYCF}-\mathrm{F}$, are used to prepare the $180^{\circ} \mathrm{C}$ resisting high temperature seawater-based fracturing fluid. With the formula of $1.0 \% \mathrm{HYCF}-5+2.5 \% \mathrm{HYCF}-\mathrm{S}+0.2 \% \mathrm{HYCF}-\mathrm{C}+0.5 \% \mathrm{HYCF}-$ $\mathrm{D}+0.2 \% \mathrm{HYCF}-\mathrm{F}+0.5 \% \mathrm{HYAS}-1+0.6 \% \mathrm{HYCF}-\mathrm{B}$, the high temperature seawater-based fracturing fluid meets the standard of oil and gas industry SY/T 6376-2008 General Technical Specifications of Fracturing Fluids in terms of its performance indexes. Through evaluation on the structure and property of HYCF-5, and properties of high temperature seawater-based fracturing fluid and the gel breaker for high temperature seawater-based fracturing fluid, the advantages and characteristics of high temperature seawater-based fracturing fluid are demonstrated from theoretical study to applied research, thereby providing sufficient theoretical support and technical reference for field application.

\section{References}

[1] ZHANG Danian, ZHAO Chongzhen, FAN Lingxiao,et al. Rapid Preparation and performance evaluation of a seawater-based vegetable gum fracturing liquid[J].China Offshore Oil and Gas,2016,28(6):95-98.

[2] Majid A B A, Hansen J E, Al-Dahlan M N, et al. Seawater Based Fracturing Fluid: A Game Changer in Hydraulic Fracturing Applications in Saudi Arabia[C].SPE Middle East Oil \& Gas Show and Conference, 2017.

[3] Prakash C, Raykov T, Kolasa B, et al. Successful Application of Seawater-Based Clean Fracturing Fluid[C]. SPE Asia Pacific Hydraulic Fracturing Conference, 2016.

[4] Wilson A. A Comparison Between Seawater-Based and Freshwater-Based Fracturing Fluids [J]. Journal of Petroleum Technology, 2017, 69(3):46-47.

[5] LIU Gangzhi, WANG Xingzun, BAO Wenhui,et al. Research on Seawater-based Fracturing Fluid Technology[J].DFCF,2013,30(3):73-75.

[6] LIN Bo,LIU Tongyi,Chen Guangjie. The Formulation and Properties of on a Seawater-Based Clean Fracturing Fluid[J].Oilfield Chemistry,2015,32(3):336-340.

[7] ZHANG Fuming,LI Xuejun,CHEN Xiaohua,et al.Preparation and Properties of Seawater-based Clean Fracturing Fluid PA-VES90[J].Science Technology and Engineering,2012,12(8):1760-1764.

[8] LI Jianbo,et al. Preparation and field application of oil field chemicals. Chemical Industry Press,2012 
[9] CONG Lianzhu, CHEN Jinfu, LI Zhiping,et al. Adsorption features of fracturing fluid by coal substrate during the fracturing process in coalbed methane well[J].Coal Geology \& Exploration,2007,35(5):27-30.

[10] WEN Qingzhi, GAO Jinjian, LIU Hua,et al. Dynamic experiment on slick-water prop-carrying capacity[J].Oil Drilling \& Production Techology,2015(2):97-100.

[11] ZHANG Huali, ZHOU Jidong, GAO Chun,et al. The damage of guar gum fracturing fluid [J]. Science Technology and Engineering,2013,13(23):6866-6871.

[12] TAO Hongsheng, WANG Manxue, XING Yi,et al. Relationship between suspended proppant ability and viscoelasticity of clean fracturing fluid with low viscosity[J].Oilfield Chemistry,2015,32(4):494-498.

[13] LEI Qiaohui, TIAN Genlin, ZHENG Dewen,et al. A study on shear loss of polymers and its restoration[J].Journal of Xi'an Shiyou University,1997(6):36-38.

[14] GUO Jianchun,HE Chunming. Microscopic mechanism of the damage caused by gelout process of fracturing fluids[J].Acta Petrolei Sinica,2012,33(6):1018-1022.

[15] YUAN Fei, SHEN Jinwei,MA Wanzheng,et al.Study on the Formation Damage Mechanisms of Guanidine Gum Fracturing Fluid to Toutun River Formation of Fu Dong Slope Area[J]. Oilfield Chemistry, 2014,31(1):42-46. 